WHAT REMAINS 



\title{
WHAT REMAINS
}

Bringing America's Missing

Home from the Vietnam War

\section{SARAH E. WAGNER}

\author{
II \\ Harvard University Press \\ Cambridge, Massachusetts \\ London, England \\ 2019
}


Copyright () 2019 by the President and Fellows of Harvard College All rights reserved

Printed in the United States of America

First printing

Cover design: Annamarie McMahon Why

Cover image: kevinjeon00 (C) Getty Images

$$
\begin{gathered}
9780674243613 \text { (EPUB) } \\
9780674243620 \text { (MOBI) } \\
9780674243606 \text { (PDF) }
\end{gathered}
$$

The Library of Congress has cataloged the printed edition as follows:

Names: Wagner, Sarah E., author.

Title: What remains : bringing America's missing home from the Vietnam War/ Sarah E. Wagner.

Description: Cambridge, Massachusetts : Harvard University Press, 2019. I Includes bibliographical references and index.

Identifiers: LCCN 2019014450 I ISBN 9780674988347 (alk. paper)

Subjects: LCSH: Vietnam War, 1961-1975-Missing in action-United States. I

Missing in action-Vietnam-Identification. I Vietnam War, 1961-1975-

Repatriation of war dead-United States. I Vietnam War, 1961-1975-Search and rescue operations-United States. I Grief-United States.

Classification: LCC DS559.8.M5 W34 2019 | DDC 959.704 / 38-dc23

LC record available at https://lccn.loc.gov/2019014450 
- TO THOSE WHO CAME HOME RIGHT AWAY

$\checkmark$ TO THOSE WHO CAME HOME YEARS LATER

+ TO THOSE WHO MAY NEVER COME HOME 
